https://creativecommons.org/licenses/by/4.0/

\title{
DESARROLLO DE LA METODOLOGÍA LEAN HEALTHCARE EN EL SERVICIO DE MEDICINA INTERNA DE UNA INSTITUCIÓN HOSPITALARIA DE CARÁCTER PÚBLICO
}

\author{
An implementation of lean healthcare methodology \\ in the internal medicine service of a public hospital
}

\author{
KAREN ANDREA AMADO LÓPEZ ${ }^{1}$, MARÍA PAULA OSORIO RUBIO ${ }^{2}$, \\ DR. ELKIN ANTONIO MOLINA GÓMEZ ${ }^{3}$, ING. EDGAR LEONARDO DUARTE FORERO ${ }^{4}$ \\ Recibido: 22 de octubre de 2019. Aceptado: 22 de diciembre de 2019 \\ DOI: http://dx.doi.org/10.21017/rimci.2020.v7.n13.a74
}

\begin{abstract}
Resumen
El uso de las metodologías Lean en el sector salud conlleva importantes mejoras en el plano operacional, así como en la cultura organizacional de instituciones hospitalarias. Este artículo presenta el desarrollo de Lean Healthcare en el Hospital Universitario de La Samaritana (HUS), una institución pública de tercer nivel de complejidad que funciona en la ciudad de Bogotá, Colombia. Se presenta una propuesta de metodología que ha sido validada en distintas áreas del hospital y que en este caso tuvo aplicación en el servicio de medicina interna, en donde se presenta una gran complejidad operativa en la toma de decisiones. Como resultado fueron planteadas oportunidades de reducir el tiempo de ciclo del plan de egresos en un 37 por ciento con medidas que podrían implementarse en el corto plazo y con una baja inversión de capital. También se encontró que, contrario a preconcepciones existentes, el uso de oxígeno domiciliario no es la causa por la cual se generan las estancias prolongadas.
\end{abstract}

Palabras clave. Lean healthcare; gestión de operaciones; medicina interna; simulación; VSM.

\begin{abstract}
Using Lean methodologies in the healthcare field draws important improvements in the operational scope. This paper presents an imlementation of Lean Healthcare at Hospital Universitario de La Samaritana (HUS), a public institution of high complexity located at Bogotá, Colombia. A proposal of methodology is provided, that has been validated in different areas of the hospital. In this case, it has been implemented in the service of internal medicin service. A reduction in the cycle time of the expenditure plan of 37 percent was achieved with short time implementation tools. Further conclusions about the training of staff are provided as an output of the process. It was also found that the use of household oxygen was not the cause of extended stays in the hospital.
\end{abstract}

Key words. Lean healthcare; operations management; internal medicine; simulation; VSM.

1 Ingeniería industrial, Estudiante de la Faculta de Ingeniería, Universidad Libre Bogotá - Colombia. Correo Electrónico: karenaamadol@unilibre.edu.co

2 Ingeniería industrial, Estudiante de la Faculta de Ingeniería, Universidad Libre Bogotá - Colombia. Correo Electrónico: mariap.osorior@unilibrebog.edu.co

3 Profesor de la Facultad de Ingeniería, Universidad Libre Bogotá - Colombia. Correo Electrónico: edgarl.duartef@unilibre.edu.co

4 Subdirector de Medicina, Hospital Universitario de la Samaritana - Colombia. Correo Electrónico: hospital.subdiretor@hus.org.co 


\section{INTRODUCCIÓN}

L

A METODOLOGÍA LEAN es una de las herramientas con mayor nivel de aceptación en el sector servicios, cuando de mejoramiento de la eficiencia se trata. Esta metodología puede definirse como "el proceso de mejorar de forma constante y gradual las diferentes áreas de una empresa, buscando una mayor productividad y competitividad de esta" [1][2]. Esta metodología se ha ido desarrollando para diversas aplicaciones que no solo se usan en la manufactura sino también en los servicios como en los gobiernos (Lean Government), los hoteles (Lean Hotel) y la contabilidad (Lean Accounting) [3].

La metodología Lean se aplica exitosamente también en el área de salud con el nombre de Lean Healthcare (Hospitales ágiles), buscando mejorar procesos, y eliminando las prácticas desperdiciadoras (Ver Tabla I) que existen en casi cualquier proceso [3].

Para lograr estos cambios significativos la organización debe contar con una combinación exitosa de esfuerzos como: visión y liderazgo, motivación, conocimientos y habilidades, planes y seguimientos, tiempo y recursos y mentalidad ganadora[3].

En el caso específico de esta investigación, Lean Healthcare fue utilizada para mejorar la gestión de operaciones logísticas en el servicio de medicina interna en el área de Hospitalización del HUS con el fin de reducir las estancias prolongadas causadas por complicaciones administrativas en la etapa del plan de egreso.

Es por eso, que se estudió el proceso desde se genera un egreso hasta que se da la orden de salida, mediante la utilización de una herramienta denominada Value Stream Mapping (VSM) y un análisis histórico de los egresos.

\section{Antecedentes}

El incremento del nivel de vida, la educación de las personas y el fácil acceso a la información a través de Internet, han elevado las exigencias en la calidad de los servicios de salud [5][6]. Una de las mayores preocupaciones en la gestión de ope-
Tabla I. Ejemplos de desperdicios en el sector salud.

\begin{tabular}{|c|c|}
\hline $\begin{array}{l}\text { Desperdicios } \\
\text { originales }\end{array}$ & $\begin{array}{c}\text { Ejemplos de desperdicios en salud } \\
\text { (Nhsiii, 2007) }\end{array}$ \\
\hline Transporte & $\begin{array}{l}\text { - Personal que camina hasta el otro } \\
\text { extremo de un área para recoger } \\
\text { notas } \\
\text { - Almacenes centrales para artículos de } \\
\text { uso común, en lugar de localizarlos } \\
\text { en almacenes de artículos de mayor } \\
\text { frecuencia de uso }\end{array}$ \\
\hline Inventario & $\begin{array}{l}\text { - Exceso de existencias en almacenes } \\
\text { que no están siendo utilizadas } \\
\text { - Pacientes en espera de ser dados de } \\
\text { alta } \\
\text { - Listas de espera }\end{array}$ \\
\hline Movimiento & $\begin{array}{l}\text { - Movimiento innecesario de personal } \\
\text { buscando papeles } \\
\text { - No contar con el equipo básico en } \\
\text { cada examen/habitación }\end{array}$ \\
\hline $\begin{array}{l}\text { En espera } \\
\text { (retardo) }\end{array}$ & $\begin{array}{l}\text { - Pacientes, resultados, preceptos y } \\
\text { medicamentos } \\
\text { - Médicos para dar alta a los pacientes }\end{array}$ \\
\hline Sobreproducción & $\begin{array}{l}\text { - Solicitud de pruebas diagnósticas } \\
\text { innecesarias }\end{array}$ \\
\hline $\begin{array}{c}\text { Durante el } \\
\text { procesamiento }\end{array}$ & \begin{tabular}{|l} 
- \\
- Puplicación de la información información sobre los pacientes \\
varias veces
\end{tabular} \\
\hline Defectos & $\begin{array}{l}\text { - Reingreso de pacientes por fallas en } \\
\text { la atención } \\
\text { - Repetición de pruebas ya que la } \\
\text { información no era correcta }\end{array}$ \\
\hline
\end{tabular}

Fuente: Los autores información suministrada de Radnor Z, Holweg M, Waring J. [4]

raciones en salud son las estancias hospitalarias prolongadas. Éstas generan efectos negativos en el sistema de salud como aumento en los costos, saturación de las urgencias y riesgos de eventos adversos.

Diferentes investigaciones han demostrado que las estancias prolongadas están dadas por factores asociados a (Ver Tabla II): personal de salud, administración hospitalaria, pacientes y relaciones entre las entidades de la red de atención [7].

En atención a esto y según el grupo de investigación de Advisory Board Company, los hospitales que logran reducir las estancias prolongadas aumentan así la capacidad efectiva como se muestra en la Tabla III. Es por esta razón, que los hospitales buscan reducir estas estancias a través de diferentes métodos como la mejora continua.

Rev. Ingeniería, Matemáticas y Ciencias de la Información Vol. 7 / Núm. 13 / enero - junio de 2020; pág. 45-56 
Tabla II. Factores que prologan la estancia hospitalaria

\begin{tabular}{|c|c|c|}
\hline Atribución & Factor & Porcentaje \\
\hline \multirow{6}{*}{$\begin{array}{l}\text { Personal de } \\
\text { salud }\end{array}$} & $\begin{array}{l}\text { No prestar atención a la } \\
\text { necesidad de dar el alta }\end{array}$ & $26,5 \%$ \\
\hline & $\begin{array}{l}\text { Falta de diagnóstico claro al } \\
\text { ingreso }\end{array}$ & $17,6 \%$ \\
\hline & Admisión prematura & $17,6 \%$ \\
\hline & $\begin{array}{l}\text { Tratamiento } \\
\text { conservador }\end{array}$ & $5,9 \%$ \\
\hline & $\begin{array}{l}\text { Técnica quirúrgica que puede } \\
\text { mejorar }\end{array}$ & $2,9 \%$ \\
\hline & $\begin{array}{l}\text { Médicos mantienen pacientes en } \\
\text { el hospital por ganancias } \\
\text { monetarias }\end{array}$ & $2,9 \%$ \\
\hline \multirow{3}{*}{$\begin{array}{l}\text { Relación entre } \\
\text { entidades de la } \\
\text { red de atención }\end{array}$} & $\begin{array}{l}\text { Necesidad de atención en otro } \\
\text { nivel de complejidad }\end{array}$ & $38,2 \%$ \\
\hline & $\begin{array}{l}\text { Procedimientos diagnósticos o } \\
\text { terapéuticos que pueden } \\
\text { realizarse ambulatoriamente }\end{array}$ & $32,4 \%$ \\
\hline & $\begin{array}{l}\text { Demora en las autorizaciones } \\
\text { por parte del asegurador }\end{array}$ & $8,8 \%$ \\
\hline \multirow{3}{*}{$\begin{array}{c}\text { Administración } \\
\text { hospitalaria }\end{array}$} & $\begin{array}{l}\text { Demora en la realización de } \\
\text { procedimientos quirúrgicos y } \\
\text { diagnósticos }\end{array}$ & $67,6 \%$ \\
\hline & Día de la semana & $29,4 \%$ \\
\hline & $\begin{array}{l}\text { Transferencia de pacientes entre } \\
\text { unidades }\end{array}$ & $2,9 \%$ \\
\hline \multirow{4}{*}{ Paciente } & Edad & $38,2 \%$ \\
\hline & Situación sociofamiliar & $38,2 \%$ \\
\hline & Condición clínica del paciente & $32,4 \%$ \\
\hline & Sexo & $11,8 \%$ \\
\hline Otro & Ocurrencia de evento adverso & $20,6 \%$ \\
\hline
\end{tabular}

Fuente: Los autores información suministrada de CeballosAcevedo T, Velásquez-Restrepo PA, Jae?n-Posada JS, 2014[7].

En la Tabla IV se enuncian algunas investigaciones que recurrieron al uso de herramientas lean para obtener mejoras notables en los procesos de la salud. Está tabla permite observar la metodología usada, la finalidad y las conclusiones en cada una de las investigaciones.
Tabla III. Ganancia "efectiva" de camas a partir de la reducción de la duración de la estancia hospitalaria.

\begin{tabular}{|c|c|c|c|c|c|}
\hline \multicolumn{5}{|c|}{ Tamaño del hospital (camas) } \\
\hline $\begin{array}{c}\text { Duración de } \\
\text { la } \\
\begin{array}{c}\text { internación } \\
\text { Reducción } \\
\text { (días) }\end{array}\end{array}$ & $\begin{array}{c}\mathbf{2 0 0} \\
\text { camas }\end{array}$ & $\begin{array}{c}\mathbf{3 0 0} \\
\text { camas }\end{array}$ & $\begin{array}{c}\mathbf{4 0 0} \\
\text { camas }\end{array}$ & $\begin{array}{c}\mathbf{5 0 0} \\
\text { camas }\end{array}$ & $\begin{array}{c}\mathbf{6 0 0} \\
\text { camas }\end{array}$ \\
\hline $\mathbf{0 , 2 5}$ días & 8 & 12 & 16 & 20 & 25 \\
\hline $\mathbf{0 , 5 0}$ días & 16 & 25 & 33 & 41 & 49 \\
\hline $\mathbf{0 , 7 5}$ días & 25 & 37 & 49 & 61 & 74 \\
\hline $\mathbf{1 , 0 0}$ día & 33 & 49 & 65 & 82 & 98 \\
\hline $\mathbf{1 , 2 5}$ días & 41 & 61 & 82 & 102 & 123 \\
\hline $\mathbf{1 , 5 0}$ días & 49 & 74 & 98 & 123 & 147 \\
\hline
\end{tabular}

Fuente: Los autores información suministrada de Tell W, Nataraja S, 2010[8].

\section{ESTRATEGIA METODOLÓGICA}

Esta investigación se realizó aplicando la metodología Lean Healthcare, la cual ha sido utilizada exitosamente en el sector salud por instituciones como: Hospital Vall D' Hebrón, [17], Hospital Universitario Virgen del Rocío de Sevilla [17] y por autores como Martin et al [18], Afsar-Manesh et al [19], para el desarrollo de procesos de mejora continua, desarrollando una cultura hacia una organización más eficiente, con el fin de incrementar la velocidad de respuesta por medio de reducción de desperdicios, costos y tiempos.

En la Fig. 1 se representa el paso a paso utilizado en el proceso del desarrollo del proyecto el cual permite evidenciar como se realizó la investigación y a qué herramientas se emplearon.

De manera preliminar, es importante aclarar que, para esta investigación, el concepto de "servicios" hospitalarios se refiere a aquellas especialidades

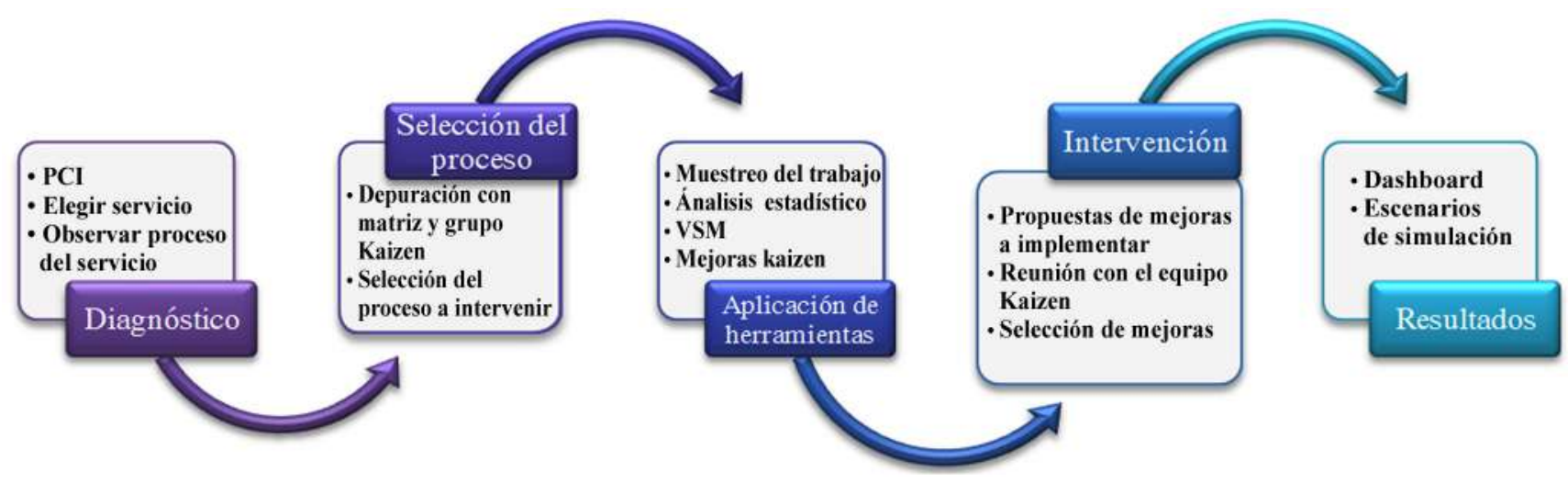

Fig. 1. Proceso metodológico[20][21]

Rev. Ingeniería, Matemáticas y Ciencias de la Información

Vol. 7 / Núm. 13 / enero - junio de 2020; pág. 45-56 
Tabla IV. Matriz de antecedentes.

\begin{tabular}{|c|c|c|c|}
\hline Autores & Planteamiento & Conclusiones & $\begin{array}{c}\text { Metodología } \\
\text { utilizada }\end{array}$ \\
\hline $\begin{array}{l}\text { Lynn D. Martin, } \\
\text { Sally E. } \\
\text { Rampersad, } \\
\text { Daniel K.-W. Low, } \\
\text { Mark A. Reed }\end{array}$ & $\begin{array}{l}\text { Transformar el sistema y la } \\
\text { cultura mediante el com- } \\
\text { promiso del personal de } \\
\text { asistencia para el desarrollo } \\
\text { de un servicio que mejora } \\
\text { continuamente y permite } \\
\text { obtener mejores resultados } \\
\text { clínicos duraderos }\end{array}$ & $\begin{array}{l}\text { Se redujeron los tiempos de espera en cirugía } \\
\text { disminuyendo los pasos que no agregaron valor } \\
\text { y a su vez los costos, mejorando la problemática y } \\
\text { alcanzando el objetivo trazado. }\end{array}$ & $\begin{array}{l}\text { Modelo CPI (Mejora- } \\
\text { miento Continuo de } \\
\text { Desempeño), Mapas } \\
\text { de secuencia de valor, } \\
\text { Casa de Toyota }\end{array}$ \\
\hline $\begin{array}{l}\text { Paloma Martínez } \\
\text { Sánchez, José Luis } \\
\text { Martínez, Judith } \\
\text { Cavazos, José } \\
\text { Pablo Nuño }\end{array}$ & $\begin{array}{l}\text { Fortalecer la percepción de } \\
\text { valor para los usuarios de } \\
\text { la IPS localizada en La } \\
\text { Sabana de Bogotá }\end{array}$ & $\begin{array}{l}\text { El uso de las herramientas VSM y el diagrama de } \\
\text { spaghetti permite a las IPS visualizar mudas o } \\
\text { desperdicios, referentes a procesos y recorridos } \\
\text { que no agregan valor o que deben disminuirse } \\
\text { con el fin de mejorar la prestación del servicio al } \\
\text { paciente }\end{array}$ & $\begin{array}{l}\text { Entrevistas, diagrama } \\
\text { de procesos, VSM, } \\
\text { diagrama de } \\
\text { spaguetti }\end{array}$ \\
\hline $\begin{array}{l}\text { Ruiz Cubillos, } \\
\text { Sonia Natalia. } \\
\text { Villarreal Anamá, } \\
\text { Judith Vanessa }\end{array}$ & $\begin{array}{l}\text { Desarrollar la metodología } \\
\text { Lean Healthcare, como } \\
\text { estrategia de mejoramiento } \\
\text { continuo, que permita ele- } \\
\text { var la eficiencia y calidad } \\
\text { del servicio prestado en el } \\
\text { área de Imágenes Diagnós- } \\
\text { ticas del Hospital Universi- } \\
\text { tario de La Samaritana } \\
\text { (HUS) }\end{array}$ & $\begin{array}{l}\text { Luego de aplicar la metodología Lean Healthcare } \\
\text { se evidenció que esta contribuye en un entorno } \\
\text { de mejora continua, ya que brinda soluciones a } \\
\text { oportunidades de mejora encontradas en el sector } \\
\text { sanitario, para el caso del proyecto, en el área de } \\
\text { Imágenes Diagnósticas del HUS. Así mismo, } \\
\text { permitió elevar la eficiencia y calidad del nivel de } \\
\text { servicio prestado. Su aplicación no fue posible sin } \\
\text { el trabajo en equipo de todo el personal del área, } \\
\text { y la retroalimentación de áreas interrelacionadas } \\
\text { como urgencias, hospitalización y enfermería. }\end{array}$ & $\begin{array}{lr}\text { VSM, 5's, } & \text { SMED, } \\
\text { poka joke, eventos } \\
\text { Kaizen, } & \text { DMAIC, } \\
\text { Herzberg }\end{array}$ \\
\hline $\begin{array}{c}\text { Laura Rocio } \\
\text { Poveda Duarte }\end{array}$ & $\begin{array}{l}\text { Estructurar un plan de } \\
\text { mejoramiento detallado } \\
\text { para el proceso de egreso } \\
\text { de pacientes del piso de } \\
\text { hospitalización de la } \\
\text { fundación cardiovascular } \\
\text { de Colombia }\end{array}$ & $\begin{array}{l}\text { El proceso de egreso está desarrollando activi- } \\
\text { dades que lo hacen improductivo, lento, ya que } \\
\text { no añaden valor para el cliente. El principal } \\
\text { desperdicio encontrado fue el tiempo de esperas. } \\
\text { Encontraron deficientes sistemas de comuni- } \\
\text { cación, procesos no estandarizados, falta de } \\
\text { capacitación y compromiso. Se requiere inver- } \\
\text { sión, pero las ganancias a mediano plazo superan } \\
\text { el coste inicial. }\end{array}$ & $\begin{array}{l}\text { Conformaron un } \\
\text { equipo LEAN, Mapa } \\
\text { de flujo de valor, } \\
\text { Diagrama de Ishika- } \\
\text { wa, Análisis de cifras, } \\
\text { Costo - beneficio }\end{array}$ \\
\hline $\begin{array}{l}\text { S. Pineda Dávila * } \\
\text { y J. Tinoco } \\
\text { González }\end{array}$ & $\begin{array}{l}\text { Evaluar si la aplicación de } \\
\text { la metodología Lean a un } \\
\text { servicio de rehabilitación } \\
\text { disminuye los derroches } \\
\text { (mudas en la terminología } \\
\text { Lean) y agrega valor al } \\
\text { cliente. }\end{array}$ & $\begin{array}{l}\text { Con la implantación de la herramienta } 5 S \text { se } \\
\text { consiguieron eliminar elementos no necesarios y } \\
\text { se estandarizó la ubicación del material, de forma } \\
\text { que mejoró el orden, la limpieza y la seguridad. } \\
\text { Se evaluaron los resultados obtenidos durante un } \\
\text { año, de julio de } 2011 \text { a julio de } 2012 \text {. Se observó } \\
\text { que la valoración del check list aumentó en los } 2 \\
\text { centros de un } 50 \% \text { y } 56 \% \text { a un } 77 \% \text { y } 74 \% \\
\text { respectivamente. }\end{array}$ & Kanban, 5S y 2P. \\
\hline $\begin{array}{l}\text { F. Boronat a, A. } \\
\text { Budiaa, E. } \\
\text { Brosetaa, J.L. } \\
\text { Ruiz-Cerdáa, D. } \\
\text { Vivas-Consuelo B. }\end{array}$ & $\begin{array}{l}\text { Descripción de la aplica- } \\
\text { ción de la metodología } \\
\text { Lean como método de } \\
\text { mejora continua de la } \\
\text { eficiencia en un servicio de } \\
\text { urología de un hospital } \\
\text { terciario. }\end{array}$ & $\begin{array}{l}\text { La aplicación de esta metodología se tradujo en } \\
\text { una alta satisfacción de los profesionales, una } \\
\text { mejora de los indicadores de calidad alcanzando } \\
\text { en } 4 \text { años un ICAR de } 0,59 \text { y un IMAR de } 0,24 \text {. En } \\
\text { el indicador de eficiencia IEAR se alcanzó un } \\
\text { valor de } 0,61 \text {, con un ahorro de } 2.869 \text { estancias } \\
\text { frente al Benchmarking nacional (IASIST). El } \\
\text { IRAR fue el único indicador por encima del } \\
\text { estándar, con un valor de } 1,36 \text {, pero con una } \\
\text { mejora evolutiva anual del mismo. }\end{array}$ & $\begin{array}{l}\text { Formación de equipo } \\
\text { y mejora del feedback } \\
\text { entre los profesiona- } \\
\text { les, Gestión por pro- } \\
\text { cesos y súper-espe- } \\
\text { cialización, Mejora de } \\
\text { indicadores (mejora } \\
\text { continua), Cuadro in- } \\
\text { tegral de dirección de } \\
\text { sistemas sanitarios }\end{array}$ \\
\hline
\end{tabular}

Fuente: Los autores información suministrada de[9-16]. 
clínicas que se ofrecen al público, mientras que los "procesos" son los conjuntos de actividades que hacen posible la prestación de cada servicio.

En primer lugar, se decidió realizar una delimitación específica de los servicios a incluir en el estudio. Hospitalización es un área que cuenta con muchos servicios como ginecología y obstetricia, medicina interna, nefrología, neurología, ortopedia, entre otros. Se decidió en conjunto con el HUS y con el director del proyecto, realizar priorización de los servicios claves para realizar la investigación mediante un PCI (Perfil de Capacidad Interna)[22][23], obteniendo como resultado la selección del servicio de medicina interna. Los criterios de inclusión tuvieron en cuenta la gobernabilidad, disponibilidad de datos, compatibilidad con la labor clínica y respeto al uso de información confidencial.

Dentro del servicio de medicina interna, existen varios procesos que también requirieron un análisis y priorización antes de ser estudiados a fondo. Esto se realizó con el apoyo de un panel de expertos, en donde se presentó un resumen de la caracterización de cada proceso perteneciente al servicio, así como la duración aproximada de cada uno de estos. Como fruto de esta reunión se eligió el proceso de plan de egreso (Ver Fig. 2)

En tercer lugar y con el proceso de "Plan de egreso" elegido se prosiguió a realizar el muestreo del trabajo [20] en el área de facturación. La etapa de "Facturación" es la tercera fase del plan de egreso. Esta decisión se sustenta en que "Facturación" implica acciones continúas relacionadas con el egreso del paciente, mientras que otras fases como "notificación del médico" son de carácter intermitente y no tienen cercanía total con el "plan de egreso". Por eso, para esta etapa y las demás se realizó un análisis estadístico de los datos arrojados por el sistema.

Como resultado del muestreo del trabajo y como se evidencia en la Fig. 3 se obtuvo el VSM el cual es una herramienta que permite "sacar a la luz problemas" e involucrar al personal en el proceso de cambio[24-26]. Esté permitió conocer a fondo el proceso y entender completamente el flujo y las actividades que no agregan valor al proceso,

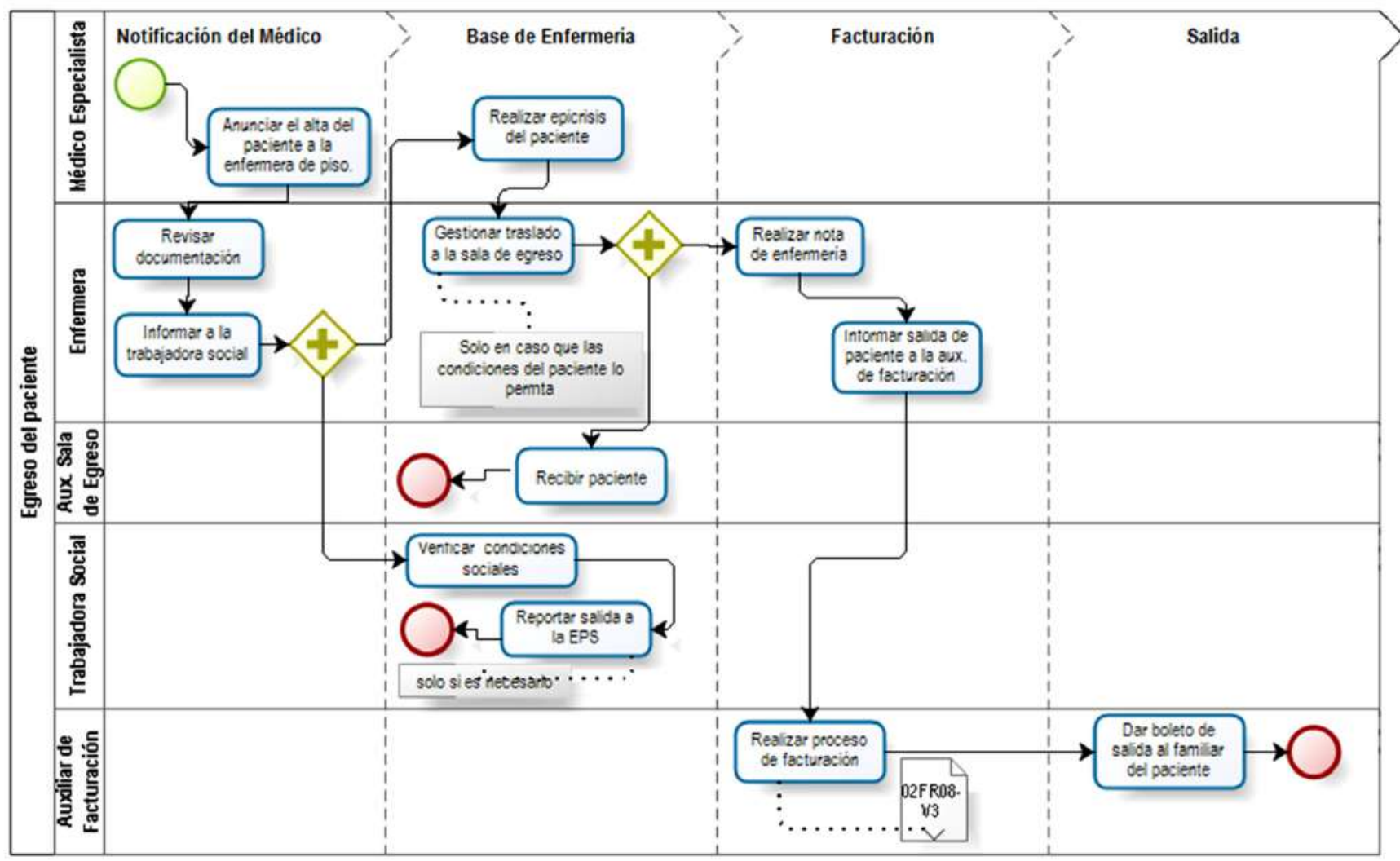

Fig. 2. Proceso del plan de egreso. 

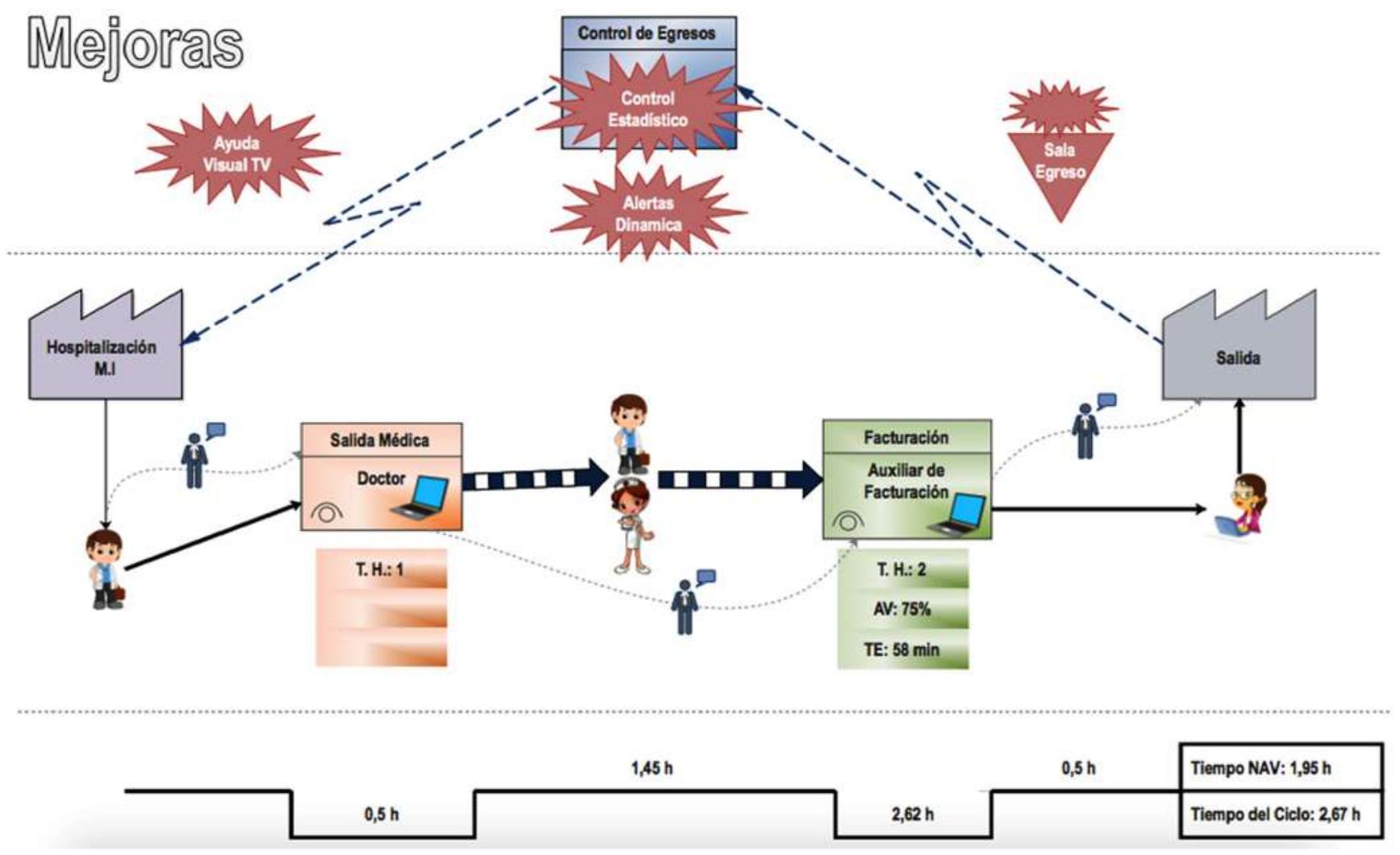

Fig. 3. VSM del proceso de plan de egreso.

permitiendo de esta manera establecer las estrategias de mejora.

En cuarto y último lugar y mediante una reunión con el equipo Kaizen "LeanKai" conformado con miembros del servicio de medicina interna se presentaron las propuestas de mejora. En esta reunión se estableció realizar un tablero de control el cual permitiera realizar una realimentación del proceso del plan de egreso y, por otro lado, realizar la evaluación de las demás mejoras a implementar a corto plazo mediante la simulación [27].

\section{Resultados}

Las mejoras propuestas fueron implementadas de la mano del equipo Kaizen del HUS. Los resultados permitieron identificar fuentes de ineficiencia, posibilidades de mejora, indicadores clave para utilizar en la toma de decisiones y mecanismos para difundir información. A continuación, se presenta una descripción de cada resultado obtenido.

\section{A. Análisis estadístico}

Se realizó un seguimiento retrospectivo de los egresos que se realizaron durante el mes de mayo y parte de junio del año 2019. Se tuvieron en cuenta atributos como la fecha de ingreso, la fecha de salida, la edad, el género, el uso de oxígeno domiciliario, la hora de salida médica, la hora de reporte en facturación y la hora de salida administrativa.

Se encontró que, contrario a una de las hipótesis planteadas por el equipo, al interior del HUS el uso del oxígeno domiciliario no es la causa por la cual se generan las estancias prolongadas. Unicamente el $20 \%$ de los 53 pacientes de medicina interna evaluados requirieron de éste.

Además, se analizó la diferencia de tiempos entre la hora de la salida médica y la llegada a facturación (diferencia uno) y entre la hora de llegada a facturación y la salida administrativa (diferencia dos). Se encontró que la diferencia uno fue mayor a la diferencia dos como se muestra en la Fig. 4 en un $48 \%$ de las veces. 


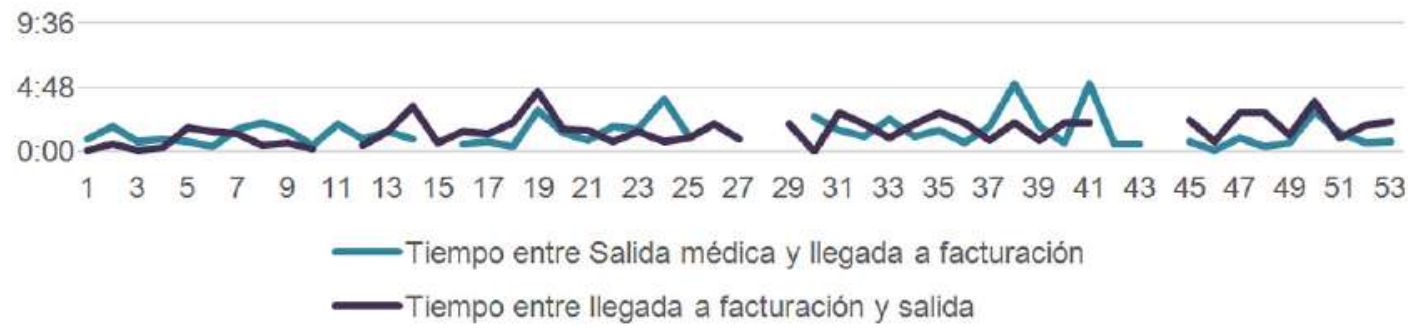

Fig. 4. Comparación de las diferencias de tiempos.

El paso de "notificación de salida a facturación" es parte del proceso del "plan de egreso", y lo desarrolla enfermería como su paso inicial. Se encontró que el $91 \%$ de las veces no se genera este aviso. Ello ocasiona demoras en el proceso de facturación el cual es muy riguroso como se puede observar en la Fig. 5, generando a su vez estancias prolongadas debido a complicaciones con el paciente que se pueden presentar durante esa demora.
Por otra parte, el uso de la sala de egreso cuando al paciente se le da de alta también es un generador de demoras. En la Fig. 6 se muestra que este uso ha disminuido entre el 2018 y el 2019. Aunque para enero de 2019 eran más los pacientes trasladados a esta sala, durante el trascurso del tiempo ha disminuido su uso en un $27 \%$ [28].

Dentro de este orden de ideas, se puede resumir que la falta de aviso previo de la salida, la

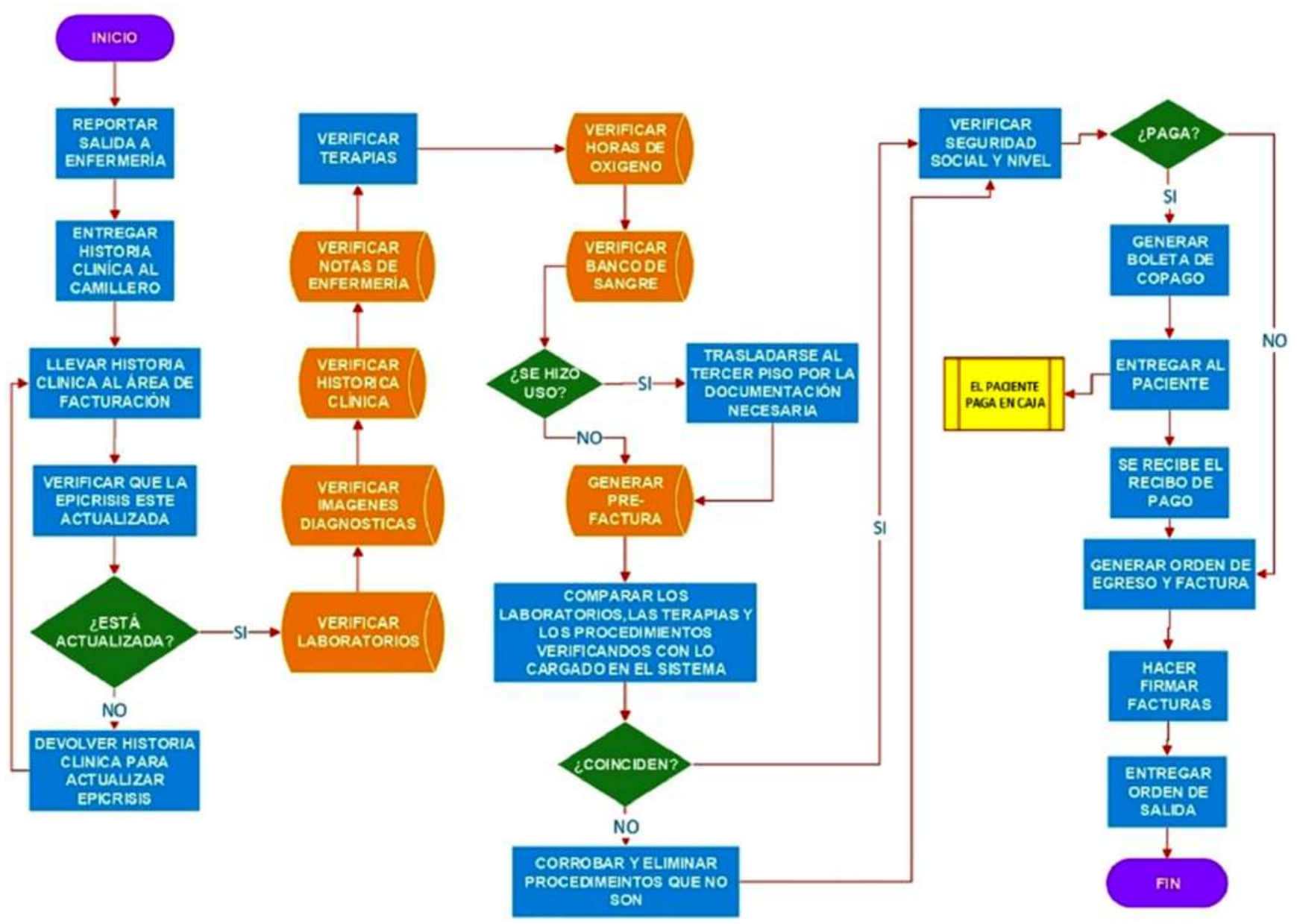

Fig. 5 Proceso de facturación 


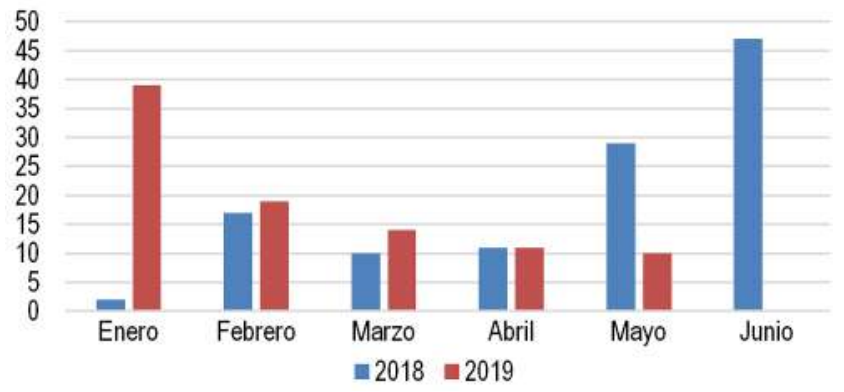

Fig. 6 Pacientes de medicina interna en sala de egresos.

demora de la llegada del egreso a facturación y la disminución del uso de la sala de egresos han generado que la estancia de los pacientes se prolongue en el servicio de medicina interna, dado que, estas demoras administrativas pueden llevar a que se generen complicaciones con el paciente.

\section{B. Tablero de control}

Como se mencionó anteriormente, una de las mejoras propuestas fue realizar una herramienta que permitiera una retroalimentación de información en el servicio de medicina interna y de esta manera buscar soluciones en los problemas presentados de acuerdo con los datos arrojados. Para este caso, se decidió realizar un Tablero de control en hoja de cálculo (Ver Fig. 7).

Este tablero muestra las demoras presentadas en la salida por mes y día de la semana. Además de ello las demoras se desglosan en tres (3) fases que permitan una evaluación detallada de las causas (Ver Fig. 8). La primera fase presenta aquellos casos cuya demora fue superior a dos (2) horas, la segunda fase muestra casos en los cuales la demora estuvo entre una (1) y dos (2) horas y la última fase muestra los casos cuya demora fue inferior a una (1) hora.

En cada una de las fases se pueden observar las demoras causadas por el proceso de facturación y las causadas por el cuerpo médico. Además, se genera una alerta que indica en dónde se encuentra la mayor demora, con el fin de que se puedan generar medidas de mejora en el área correcta (Ver Fig. 9).

El Tablero de control se alimenta de una base de datos descargada del software de gestión del HUS y se basa principalmente en las horas registradas en el sistema de cada actividad.

\section{CÁLCULO Y COONTROL DE DEMORAS EN MEDICINA INTERNA}
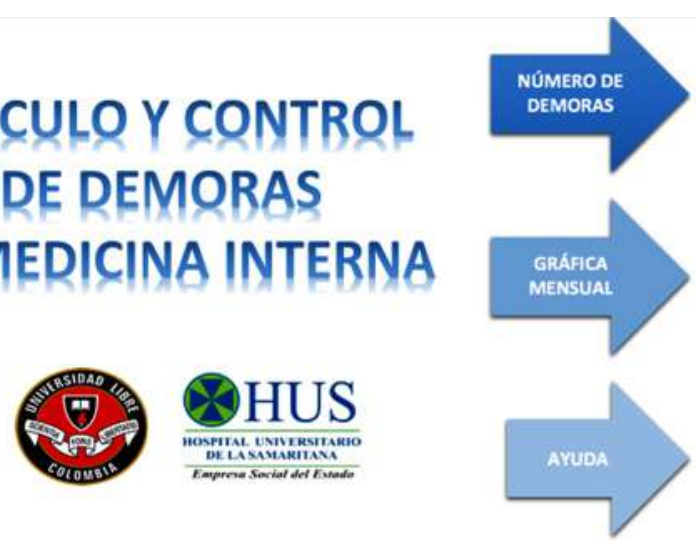

Fig. 7. Tablero de control- Inicio.

Esta herramienta permitió evaluar el tiempo de ciclo de cada una de las fases del proceso del plan de egreso de medicina interna, para así obtener y detallar las falencias que se presentan actualmente y las que se pueden seguir presentando durante el proceso. Todo esto permite buscar soluciones y realizar una toma de decisiones más acertada, basada en los datos históricos del servicio de medicina interna.

\section{Simulación discreta}

Las demás medidas de mejora que surgieron del VSM se consideraron de compleja implementación por parte del equipo Kaizen. Para validar que su implementación fuese provechosa, se decidió utilizar la simulación discreta como medio para la toma de decisiones construyendo un modelo del proceso que permitiera representar las acciones a aplicar. Las nuevas acciones evaluadas fueron la generación de alertas en el área de facturación cuando se presente un egreso, la instalación de un puesto auxiliar de facturación y cambio en el horario de los turnos de facturación.

La simulación es una técnica que permite recrear ciertos aspectos de la realidad en una computadora, con el objetivo de estudiar cómo funcionaría un determinado sistema y cómo reaccionaría frente a ciertos cambios, como ser un aumento/reducción de la demanda o capacidad de un recurso. Dado que la experimentación sobre sistemas reales resulta muy costosa y en el caso del sector de la salud podría resultar inviable ya que podría interferir con el proceso de atención, este tipo de programas presenta gran utilidad [17].

Rev. Ingeniería, Matemáticas y Ciencias de la Información Vol. 7 / Núm. 13 / enero - junio de 2020; pág. 45-56 


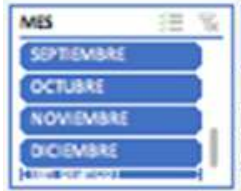

\begin{tabular}{|c|c|c|c|c|c|c|c|c|c|}
\hline \multirow[b]{2}{*}{ color } & \multirow[b]{2}{*}{-T } & \multicolumn{3}{|c|}{ DEMORA SALDA } & \multirow[b]{2}{*}{ Jueves } & \multirow[b]{2}{*}{ Viarnes } & \multirow[b]{2}{*}{ Stbado } & \multirow[b]{2}{*}{ Domingo } & \multirow[b]{2}{*}{ TOTAL } \\
\hline & & Lunes & Martes & Múrcoles & & & & & \\
\hline 0 & & 116 & 153 & 144 & 160 & 149 & 144 & 138 & 1004 \\
\hline $\mathrm{O}$ & & 26 & 25 & 24 & 17 & 19 & 13 & 20 & 144 \\
\hline 0 & & 37 & 21 & 24 & 26 & 29 & 32 & 19 & 188 \\
\hline TOTAL & & 179 & 199 & 192 & 203 & 197 & 189 & 177 & 1336 \\
\hline
\end{tabular}
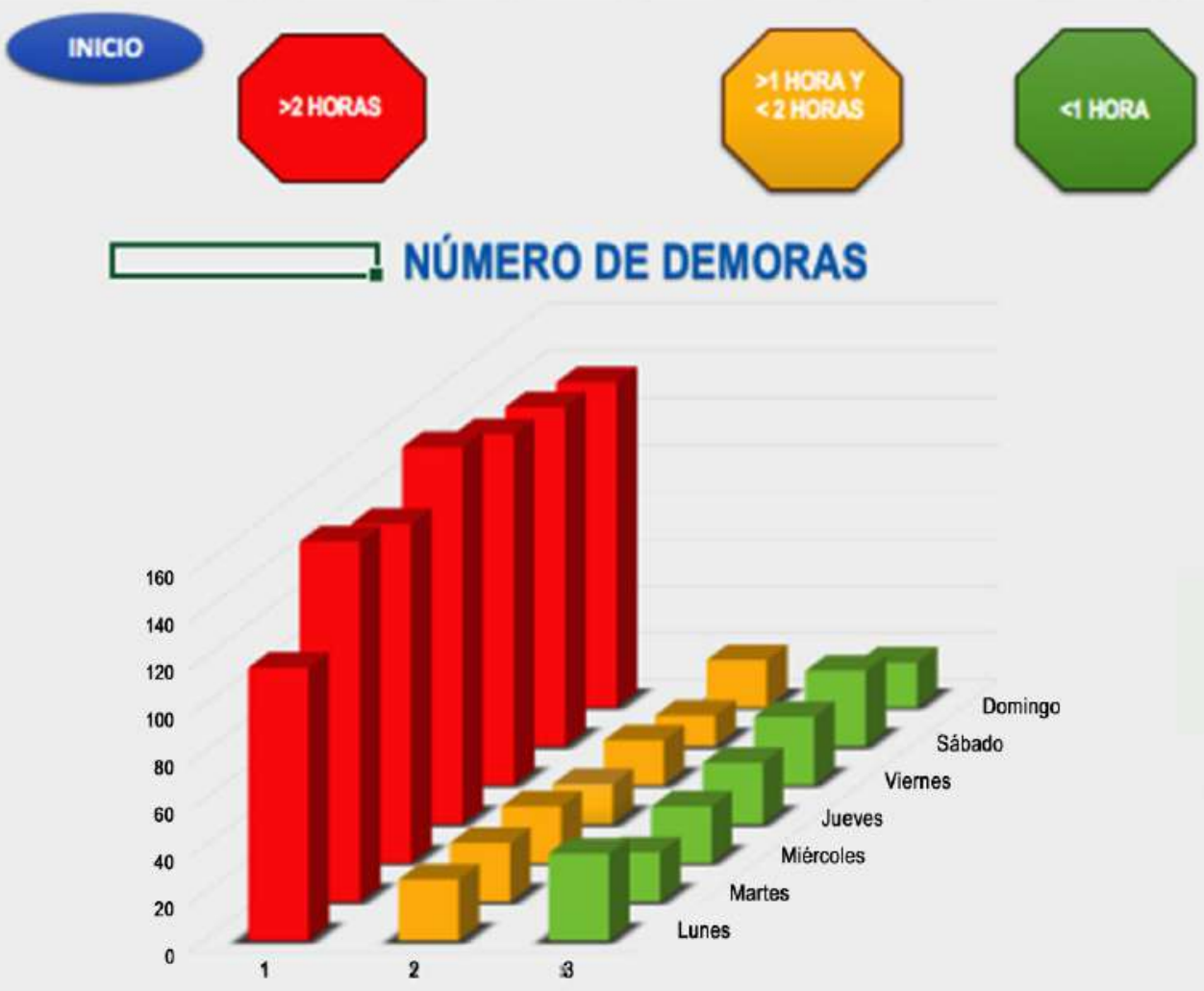

Fig. 8. Tablero de control - Número de demoras.
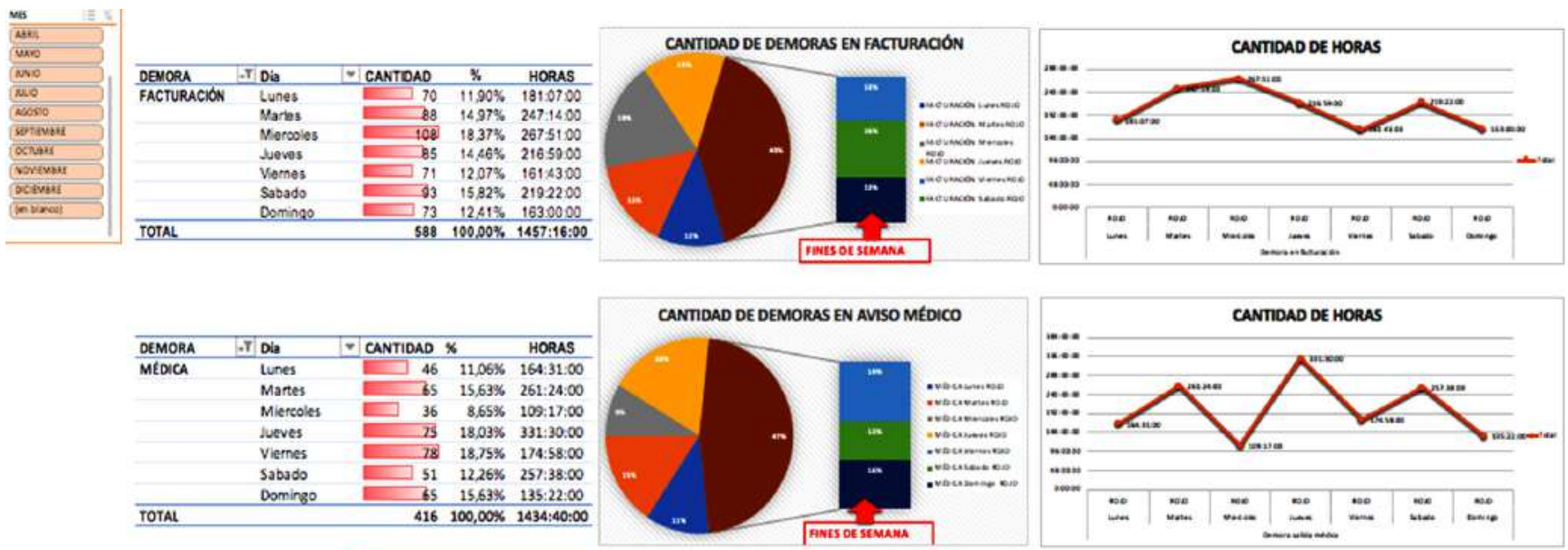

EL. MAYOR NÚMERO DE DENORAS ESTÁ EN FACTURACIÓN

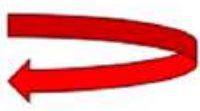

Fig. 9. Fase mayor a 2 horas. 
Es por ello por lo que se realizó una simulación estocástica la cual incorpora componentes aleatorios. La Fig. 10 representa el modelo tridimensional realizado para la simulación del proceso, utilizando el aplicativo SIMIO.

Se decidió simular el jueves de cada semana, dado que según los datos históricos es allí cuando se presentan la mayor cantidad de egresos. Para esto, se tuvieron en cuenta datos como el tiempo entre la salida médica y facturación, el tiempo en facturación y el tiempo en auditoría. Por otro lado, la duración de la salida médica se manejó como una constante dado que el análisis estadístico permitió establecer una variabilidad despreciable [18][19].

Para comprobar que el modelo se ajustaba a la realidad del proceso del plan de egreso de medicina interna se realizó una prueba de hipótesis [19] con el tiempo de ciclo del proceso arrojado por el VSM realizado y el tiempo de ciclo resultante de la simulación, encontrándose una diferencia entre ellos del seis por ciento, por lo cual, se aceptó la hipótesis nula. De esta manera se contó con la seguridad de que se contaba con una representación fidedigna del día a simular y se procedió a evaluar las mejoras mencionadas.

Dado que el $48 \%$ de las veces se presenta una demora en la llegada del reporte de salida a facturación se evaluó la generación de una alerta en el momento en que se realiza la epicrisis, es decir, que la demora entre la salida médica y la llegada a facturación se redujera. Como resultado se obtuvo un potencial de mejora del 35\%, pudiéndose disminuir el tiempo de ciclo a 3,2 horas.
La siguiente acción considerada fue evaluar el impacto que tendría aumentar el número de auxiliares de facturación para los días que se presentan más egresos, esto con el fin de disminuir el tiempo de ciclo del proceso. Se logró una mejora del $1 \%$, es decir, una disminución de 2 min en el tiempo de ciclo.

En la última mejora se consideró cambiar el horario del turno de las auxiliares de facturación, teniendo en cuenta que el mayor número de egresos se genera en las horas de la tarde. Se evaluó la simulación con un horario de 10:00 a 19:00. Se obtuvo una mejora potencial del cuatro por ciento (4\%), lo que representa una disminución de $10 \mathrm{~min}$ en el tiempo de ciclo.

Por último, la Tabla V muestra los resultados de las mejoras simuladas individualmente y combinadas.

\begin{tabular}{|} 
Tabla V. Resultados de la simulación. \\
\begin{tabular}{|c|c|c|c|}
\hline Mejoras & $\begin{array}{c}\text { Tiempo de } \\
\text { ciclo promedio } \\
\text { simulador }\end{array}$ & $\begin{array}{c}\text { Tiempo } \\
\text { disminuido }\end{array}$ & $\begin{array}{c}\text { Porcentaje } \\
\text { de mejora }\end{array}$ \\
\hline Alertas & $3,19 \mathrm{~h}$ & $1,74 \mathrm{~h}$ & $35 \%$ \\
\hline $\begin{array}{c}\text { Instalación } \\
\text { de un } \\
\text { puesto } \\
\text { auxiliar }\end{array}$ & $4,90 \mathrm{~h}$ & $2 \mathrm{~min}$ & $1 \%$ \\
\hline $\begin{array}{c}\text { Cambio de } \\
\text { horario en } \\
\text { facturación }\end{array}$ & $4,77 \mathrm{~h}$ & $10 \mathrm{~min}$ & $4 \%$ \\
\hline $\begin{array}{c}\text { Alertas - } \\
\text { Nuevo } \\
\text { puesto }\end{array}$ & $3,11 \mathrm{~h}$ & $\mathbf{1 , 8 2} \mathrm{h}$ & $37 \%$ \\
\hline $\begin{array}{c}\text { Alertas - } \\
\text { Cambio de } \\
\text { horario }\end{array}$ & $3,60 \mathrm{~h}$ & $\mathbf{1 , 3 3} \boldsymbol{h}$ & $27 \%$ \\
\hline
\end{tabular}
\end{tabular}

Fuente: Los autores.

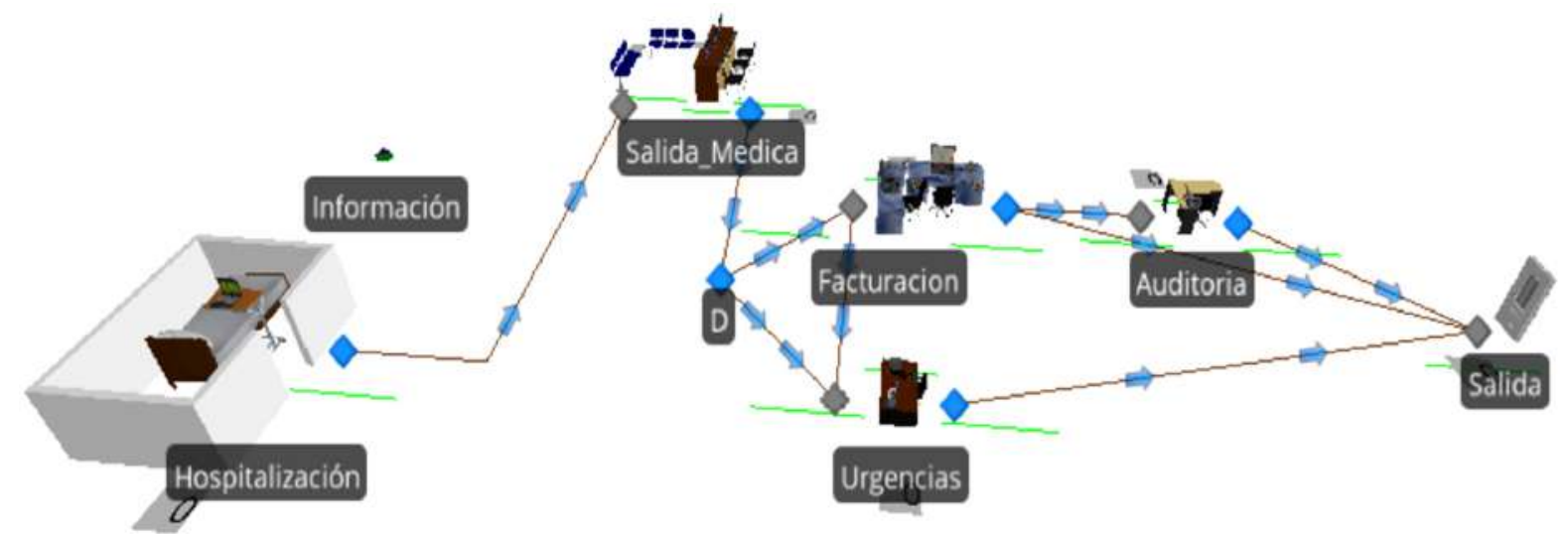

Fig. 10. Modelo del plan de egreso de medicina interna. 


\section{CONCLUSIONES Y RECOMENDACIONES}

Mientras que se desarrollaba esta investigación, fueron encontradas barreras como la resistencia del personal a la introducción de cambios, debido a que el Lean Healthcare [20] [21] conlleva la generación de nuevos hábitos y la implementación de actividades de mejora en los procesos evaluados. Por tal motivo se estudian las barreras presentadas y se les maneja de tal manera que trabajen en conjunto con el proyecto. Una manera de combatir la barrera de la resistencia del personal fue la creación de un grupo Kaizen, donde se comunicaba y explicaba lo relacionado con la metodología por medio de lúdicas.

Esta investigación permitió evidenciar la importancia que tiene el plan de egreso en el servicio de medicina interna. Este plan es crítico y poder intervenirlo representa una ayuda significativa para el hospital en cuanto a reducción de tiempos y optimización de procesos.

Por otro lado, las herramientas seleccionadas para esta aplicación (Value Stream Mapping y Tablero de control) demostraron ser de utilidad y totalmente adaptables al sector de la Salud [22]. El Tablero de control demostró la importancia que tiene crear herramientas de concentración y difusión de información dentro de un proceso. Se logró conocer de manera organizada los datos del plan de egreso y con esto poder tomar decisiones dentro del servicio.

Por otra parte, la simulación discreta permitió probar el impacto de potenciales mejoras, $\mathrm{y}$ demostrar que efectivamente se habían detectado causas raíz. Se logró una mejora alrededor del 35\% al disminuir las demoras presentadas en el egreso del paciente. Si bien la simulación fue realizada para un día en particular, las medidas planteadas no representan un gasto adicional para el hospital por lo cual es altamente recomendable que sean realizadas.

Finalmente, es necesario fomentar la formación en estos temas en las empresas e instituciones dado que permite lograr un mayor aprovechamiento de su potencial en futuras líneas de investigación que permita realizar aplicaciones concretas de esta filosofía. Como es el caso del servicio de Neurocirugía del HUS que también desarrollo Lean Healthcare y obtuvo mejoras significativas.

\section{REFERENCIAS}

[1] Sainz de Vicuña, J.M. (2002), Utilización de herramientas y técnicas de gestión en la CAPV 2001. Zamudio: SPRI

[2] Eguren, J. A., \& Goti, A. (2009). Aplicación de un Modelo de Mejora Continua en empresas auxiliares de automoción y electrodoméstico. XII Congres de Ingeniería de Organización

[3] Socconini L. (2008). Lean Manufacturing Paso a Paso. Grupo editorial Norma.

[4] Zoe Radnor \& Stephen P. Osborne (2012): Lean: A failed theory for public services?, Public Management Review, DOI:10.1080/14719037.2012.748820

[5] Smith, P. (2000). Reforming markets in health care: an economic perspective. McGraw-Hill Education (UK).

[6] González López-Valcárcel, B. (2005). Difusión de nuevas tecnologías sanitarias y políticas públicas. Masson.

[7] Ceballos-Acevedo T, Velásquez-Restrepo PA, JaénPosada JS. Duración de la estancia hospitalaria. Metodologías para su intervención. Rev. Gerenc. Polít. Salud. 2014; 13(27): 274-295. http://dx. doi.org/10.11144/Javeriana.rgyps13-27.dehm

[8] Tell W, Nataraja S. Next-Generation Capacity Management. Collaborating for Clinically Appropriate and Efficient Inpatient Throughput. Advisory Board International; 2010.

[9] Global, L., \& Fecha, N. (2014). Implantación del Lean en un hospital . Caso CSG, 19, 149-166.

[10] González Correa, F. (2007). Manufactura Esbelta (Lean Manufacturing). Principales Herramientas. Revista Panorama Administrativo, 1(2), 85-112.

[11] Martin, L. D., Rampersad, S. E., Low, D. K. W., \& Reed, M. A. (2014). Mejoramiento de los procesos en el quirófano mediante la aplicación de la metodología Lean de Toyota. Revista Colombiana de Anestesiología, 42(3), 220-228. https://doi.org/ 10.1016/j.rca.2014.02.007

[12] Martínez, P., Martínez, J., Nuño, P., \& Cavazos, J. (2015). Mejora en el Tiempo de Atención al Paciente en una Unidad de Urgencias Mediante la Aplicación de Manufactura Esbelta. Información Tecnológica, 26(6), 187-198. https:// doi.org/ 10.4067/S0718-07642015000600019

[13] Connolly, C. (3 de junio de 2005). Toyota Assembly Line Inspires Improvements at Hospital. Washington Post. Obtenido de http:/ / www.washington post.com/wpdyn/content/article/2005/06/02/ AR2005060201944.html 
[14] D' Andreamatteo, A., Ianni, L., Lega, F., \& Sargiacomo, M. (septiembre de 2015). Lean in healthcare: A comprehensive review. Health Policy, 119, 1197 - 1209. doi : http:/ /dx.doi.org/10.1016/j.healthpol. 2015.02.002

[15] Rajadell, M., \& Sánchez, J. (2010). Lean Manufacturing: la evidencia de una necesidad. España: Díaz de Santos.

[16] Ruiz Orjuela, E. T., \& Ortiz Pimiento, N. R. (diciembre de 2015). Lean Healthcare: Una revisión bibliográfica y futuras líneas de investigación. Scientia et Technica, 8. Obtenido de http://revistas. utp.edu.co/index.php/revistaciencia/article/ viewFile/11181/795 1

[17] Metodología Lean en Sanidad: Casos de éxito. (2017, noviembre 30). Recuperado 30 de julio de 2019, de MBA • IMF website: https:/ / blogs.imfformacion.com/blog/mba/exito-metodologialean-sanidad/

[18] Martin, L. D., Rampersad, S. E., Low, D. K. W., \& Reed, M. A. (2014). Mejoramiento de los procesos en el quirófano mediante la aplicación de la metodología Lean de Toyota. Revista Colombiana de Anestesiología, 42(3), 220-228. https://doi.org/ 10.1016/j.rca.2014.02.007

[19] Afsar-Manesh, N., Lonowski, S., \& Namavar, A. A. (2017). Leveraging lean principles in creating a comprehensive quality program: The UCLA health readmission reduction initiative. Healthcare (Amsterdam, Netherlands), 5(4), 194-198. https:// doi.org/10.1016/j.hjdsi.2016.12.002

[20] Hernández Sampieri, R., Fernández- Collado, C., \& Baptista Lucio, P. (2006). Metodología de la Investigación (Cuarta). México: Mc Graw Hill.
[21] Niebel, B. (2014). Ingeniería Industrial de Niebel: métodos, estándares y diseño del trabajo. México: McGraw-Hill.

[22] Rother, M., \& Shook, J. (1999). Learning to see. Boston, MA: Lean Enterprise Institute.

[23] Tapping, D., Luyster, T., \& Shulzer, T. (2003). Value Stream Management : Eight Steps to Planning, Mapping and Sustaining Lean Improvements. Journal for Healthcare Quality, 25(6), 47.

[24] Escuder, M., Tanco, M., Santoro, A (2015). Experiencia de Implementación de Lean en un Centro de Salud de Uruguay. Memoria Investigaciones en Ingeniería, núm. 13

[25] Escuder, M., Tanco, M., Santoro, A (2015). Barriers Hindering the Introduction of Lean in the Healthcare Sector. Working paper under review.

[26] Arciniégas C, L. C., Camacho O, M. Á., Duarte F, E. L., \& Naranjo L., A. (2016). Medición del desempeño de la red de suministros de medicamentos en un hospital público de tercer nivel en la ciudad de Bogotá, a través del cuadro de mando integral. INGENIARE, (20), 75. https:// doi.org/10.18041/ 1909-2458/ingeniare. 20.410

[27] Hernández-Chinchilla, D., Camacho-Oliveros, M. Á., \& Duarte-Forero, E. L. (2017). Análisis del flujo de pacientes en el servicio de urgencias del Hospital Universitario la Samaritana a través de simulación discreta. AVANCES < BR>Investigación en Ingeniería, 14, 109. https://doi.org/10.18041/ 1794-4953/avances.1.1289

[28] Otálora, L. A., Camacho, M. Á., Duarte-Forero, E. L., \& Ahumada, A. E. (2016). Evaluación de políticas de gestión de inventarios de medicamentos para un sistema multinivel y multiproducto en el Hospital Universitario de la Samaritana (HUS). INGENIARE, (21), 93-107. 